\title{
Improving gait adaptability in patients with hereditary spastic paraplegia (Move-HSP): study protocol for a randomized controlled trial
}

Lotte van de Venis ${ }^{1 *}$ (D, Bart P. C. van de Warrenburg ${ }^{2}$, Vivian Weerdesteyn ${ }^{1}$, Bas J. H. van Lith', Alexander C. H. Geurts ${ }^{1,3+}$ and Jorik Nonnekes ${ }^{1,3+}$

\begin{abstract}
Background: People with hereditary spastic paraplegia (HSP) experience difficulties adapting their gait to meet environmental demands, a skill required for safe and independent ambulation. Gait adaptability training is possible on the C-Mill, a treadmill equipped with augmented reality, enabling visual projections to serve as stepping targets or obstacles. It is unknown whether gait adaptability can be trained in people with HSP.

Aim: The aim of Move-HSP is to study the effects of ten 1-h sessions of C-Mill training, compared with usual care, on gait adaptability in people with pure HSP. In addition, this study aims to identify key determinants of C-Mill training efficacy in people with pure HSP.

Method: Move-HSP is a 5-week, two-armed, open-label randomized controlled trial with a cross-over design for the control group. Thirty-six participants with pure HSP will be included. After signing informed consent, participants are randomized (1:1) to intervention or control group. All participants register (near) falls for 15 weeks, followed by the first assessment (week 16), and, thereafter, wear an Activ8 activity monitor for 7 days (week 16). The intervention group receives 10 sessions of C-Mill training (twice per week, 1-h sessions; weeks 17-21), whereas control group continues with usual care (weeks 17-21). Afterwards, both groups are re-assessed (week 22). Subsequently, the intervention group enter follow-up, whereas control group receives 10 sessions of C-Mill training (weeks 23-27), is re-assessed (week 28), and enters follow-up. During follow-up, both groups wear Activ8 activity monitors for 7 days (intervention group: week 23, control group: week 29) and register (near) falls for 15 weeks (intervention group: weeks 23-37, control group: weeks 29-43), before the final assessment (intervention group: week 38, control group: week 44). The primary outcome is the obstacle subtask of the Emory Functional Ambulation Profile. Secondary outcomes consist of clinical tests assessing balance and walking capacity, physical activity, and fall monitoring.

(Continued on next page)
\end{abstract}

\footnotetext{
* Correspondence: lotte.vandevenis@radboudumc.nl

${ }^{\dagger}$ Alexander C. H. Geurts and Jorik Nonnekes contributed equally to this work. 'Department of Rehabilitation; Center of Expertise for Parkinson \& Movement Disorders, Donders Institute for Brain, Cognition and Behavior; Radboud University Medical Center, PO Box 9101, 6500 HB Nijmegen, The Netherlands Full list of author information is available at the end of the article
}

(c) The Author(s). 2021 Open Access This article is licensed under a Creative Commons Attribution 4.0 International License, which permits use, sharing, adaptation, distribution and reproduction in any medium or format, as long as you give appropriate credit to the original author(s) and the source, provide a link to the Creative Commons licence, and indicate if changes were made. The images or other third party material in this article are included in the article's Creative Commons licence, unless indicated otherwise in a credit line to the material. If material is not included in the article's Creative Commons licence and your intended use is not permitted by statutory regulation or exceeds the permitted use, you will need to obtain permission directly from the copyright holder. To view a copy of this licence, visit http://creativecommons.org/licenses/by/4.0/ The Creative Commons Public Domain Dedication waiver (http://creativecommons.org/publicdomain/zero/1.0/) applies to the data made available in this article, unless otherwise stated in a credit line to the data. 
(Continued from previous page)

Discussion: Move-HSP will be the first RCT to assess the effects of C-Mill gait adaptability training in people with pure HSP. It will provide proof of concept for the efficacy of gait adaptability training in people with pure HSP.

Trial registration: Clinicaltrials.gov NCT04180098. Registered on November 27, 2019.

Keywords: Hereditary spastic paraplegia, C-Mill, Gait adaptability, Rehabilitation

\section{Background}

Hereditary spastic paraplegia (HSP) is a heterogeneous group of neurodegenerative disorders, caused by retrograde axonal degeneration of the corticospinal tracts, fasciculus gracilis fibers, and to a lesser extent, the spinocerebellar fibers [1-3]. Pure forms of HSP are clinically characterized by progressive spasticity, muscle weakness, and reduced proprioception in the lower extremities, as well as difficulties in making rapid (alternating) leg movements [4-6]. Additional symptoms are present in complex forms of HSP, including mental retardation, epilepsy, ataxia, peripheral neuropathy, or optic atrophy [1, 4, 7]. For people with pure HSP, gait and balance impairments are among the most disabling symptoms. They especially experience difficulties when forced to adapt their gait to meet environmental demands, hampering the ability to walk safely and independently in the community [4, 8-11]. A recent study reported that $57 \%$ of pure HSP patients fell at least twice a year, and 73\% experience fear of falling [11]. Incorporating gait adaptability training in rehabilitation programs for people with pure HSP seems, therefore, logical and potentially beneficial $[4,11,12]$.

A limited number of task-specific gait interventions have shown to improve walking capacity in people with pure HSP. Twenty-five sessions of robot-assisted exoskeleton and overground walking improved walking velocity and balance capacity [13]. In addition, eighteen sessions of robotic Lokomat training increased walking speed, balance capacity, and quality of life [9]. Even though these results are promising, the interventions lacked tasks that promote gait adaptability. As a consequence, it remains unknown whether people with pure HSP will benefit from gait adaptability training [4]. Furthermore, it is unclear how to tailor gait rehabilitation programs to the individual patient with HSP as it is currently unknown which determinants can predict training efficacy.

To fill this gap, Move-HSP is the first randomized controlled trial to provide proof of concept for the efficacy of gait adaptability training in people with pure HSP. The training takes place in a safe environment on the C-Mill, a treadmill providing augmented reality via visual projections onto the treadmill. Participants will train obstacle negotiation, precision stepping, and unexpected accelerations and decelerations. Its feasibility and efficacy have been described in multiple neurological populations, including patients with stroke [14], cerebellar ataxia [15], and multiple sclerosis [16]. Currently, the clinical experience with gait adaptability C-Mill training for people with pure HSP is positive, but the scientific evidence is lacking [4].

\section{Objectives}

This study aims to provide an essential step towards evidence-based and individually tailored gait rehabilitation in people with HSP. The objectives are twofold:

1. To study the effect of ten 1 -h sessions of C-Mill training on gait adaptability in people with pure HSP.

2. To identify key determinants of C-Mill training efficacy in people with pure HSP.

\section{Methods/design}

\section{Regulation statement}

Move-HSP will be conducted according to the principles of the Declaration of Helsinki (64th WMA General Assembly, Fortaleza, Brazil, October 2013) and the Medical Research Involving Human Subjects Act. The protocol is written in accordance with the SPIRIT 2013 checklist.

\section{Study design and setting}

Move-HSP is a 5-week, single-center, two-armed, openlabel, randomized controlled trial (RCT), with a cross-over design for the control group, as they receive the intervention after a waiting list period. The study is conducted at the Radboud University Medical Center (Radboudumc) within the Center of Expertise for Parkinson \& Movement Disorders; Nijmegen, The Netherlands. C-Mill training can be given at the Radboudumc (Nijmegen, The Netherlands), Paramedisch centrum Rembrandt (Veenendaal, The Netherlands), Stichting Tante Louise (Bergen op Zoom, The Netherlands), and Fysiotherapie De Lindehoeve (Voorschoten, The Netherlands). Other training locations may be added while the study is ongoing, depending on the success of participant inclusion.

\section{Recruitment and selection}

Participants will be recruited at the Center of Expertise for Parkinson \& Movement Disorders of the Radboudumc (part of the European Reference Network for Rare 
Neurological Diseases (ERN-RND)). The treating physician informs the patient about Move-HSP and asks for permission whether the investigator (LV) may contact the patient. In addition, a request to participate will be sent to members of the HSP patient organization "Spierziekten Nederland". Those who are interested can contact the investigator and will receive an information letter. After 2 weeks, the investigator (LV) will contact those who expressed their interest and ask for their final decision. If patients agree to participate, eligibility is checked. After inclusion, participants can leave the study at any time without consequences.

\section{Eligibility}

For inclusion, participants will have to meet the following inclusion criteria:

- Diagnosis of pure HSP by a neurologist specialized in inherited movement disorders. Diagnosis is based on inheritance pattern and clinical examination, and when available, molecular diagnosis.

- Age between 18 and 70 years old

- Ability to walk barefoot on a level ground for $50 \mathrm{~m}$ without a walking aid (use of orthotic devices or orthopedic shoes is allowed)

Participants will be excluded if they suffer from other neurological, orthopedic, or psychiatric conditions, or if patients underwent an HSP-related surgical procedure of the lower extremities.

\section{Group allocation and blinding}

Participants will be allocated at random to the intervention group or to the (waiting list) control group following a 1:1 ratio. Randomization will be stratified based on disease duration ( 2 categories: $0-15$ years; $>15$ years) in blocks with a variable size $(n=4$ or $n=6)$ to prevent an uneven distribution between groups. To determine disease duration, participants are asked for the year of symptom onset. Randomization will be performed in CastorEDC, a web-based data management system for academic studies (www.castoredc.com). Blinding of participants is not possible, as participants will know whether they receive C-Mill training or continue with usual care. The primary investigator (LV) takes part in the training sessions as a physical therapist and, therefore, cannot be blinded either.

\section{Participant timeline}

The outline of this study is shown in Fig. 1. Following inclusion, participants are randomly allocated to either the intervention group or the control group (waiting list). During the first 15 weeks, all participants register (near) falls in a digital fall calendar. Thereafter,

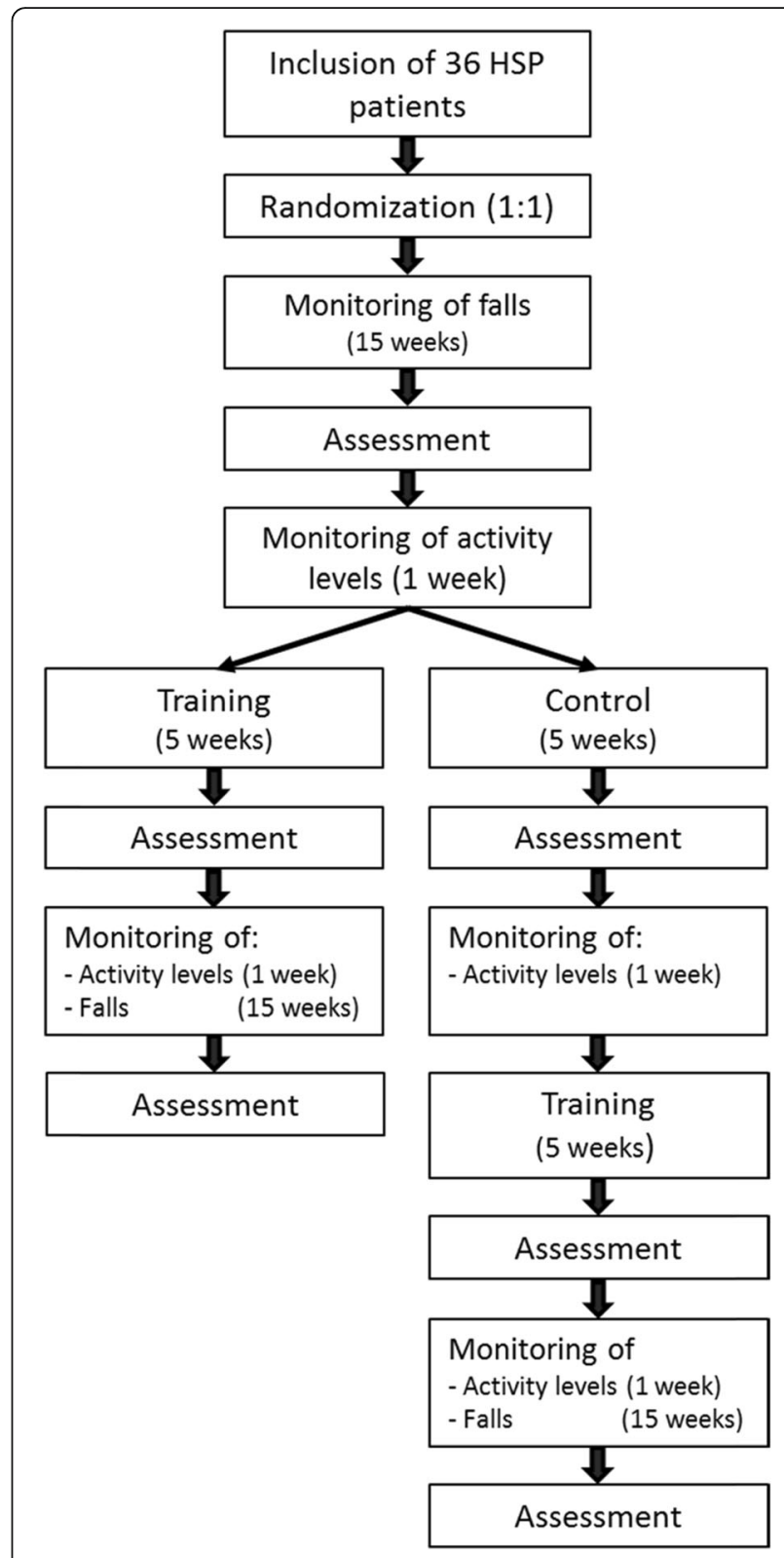

Fig. 1 Flowchart of the study protocol

participants will have the first assessment at the movement laboratory (Radboudumc; week 16). Following this assessment, participants wear an Activ8 activity monitor for seven consecutive days (week 16). Thereafter, the control group enters a waiting period of 5 weeks (weeks 17-21), whereas the intervention group starts with 5 weeks of gait adaptability training on the C-Mill (weeks 17-21). Each session lasts $1 \mathrm{~h}$ and takes place twice per week. Subsequently, both groups are reassessed (week 22). Following this second assessment, the intervention group enters the follow-up period, whereas the control group wears the Activ8 activity monitors for 7 days (week 22), starts 5 weeks of gait 
adaptability training (weeks 23-27), has the third assessment (week 28), and thereafter, enters the follow-up period. During follow-up, both groups wear Activ8 activity monitors during the first week (intervention group: week 23, control group: week 29) and, additionally, register (near) falls for 15 weeks (intervention group: weeks 23-37, control group: weeks 29-43). After followup, participants have a final assessment in the movement laboratory (intervention group: week 38 , control group: week 44).

During Move-HSP, all participants can continue their usual care. For some participants, this may include local intramuscular injections of botulinum toxin (BTX). To limit the influence of BTX injections on the outcomes, the scheduling of the assessments will consider the date of the BTX injections. BTX injections induce an effect on muscle spasticity approximately 2 weeks postinjection. The maximum effect is reached around 6-8 weeks, after which it gradually subsides $[17,18]$. Participants who receive BTX injections in the lower extremities will have the pre-intervention assessment 4 weeks post-injection, and the post-intervention assessment 10 weeks post-injection. In addition, it will be monitored whether the dosage of oral antispasmodic changes during the trial.

\section{Control group}

The eighteen participants attributed to the control group are asked to continue with their daily routine and usual care during the 5 weeks on the waiting list. If therapy is part of the usual care, participants are requested to continue with the same frequency and composition during the waiting period.

\section{Intervention: C-Mill training}

Gait adaptability training takes place on the C-Mill (Motek Medical, Culemborg, The Netherlands). The CMill is a treadmill, providing augmented reality via visual cues projected onto the treadmill. The projections are either stepping targets or obstacles that challenge the participants to adjust their steps accordingly. The training sessions take place during five consecutive weeks, twice per week during 60-min sessions. In total, participants will train gait adaptability on the C-Mill for $10 \mathrm{~h}$.

The C-Mill protocol is based on clinical experience and finalized after a focus group discussion with expert physical therapists. The training sessions are logged to ensure compatibility and a consistent progression. Each session starts with a 10-min warming-up, followed by five training blocks (Fig. 2, video). Each training block lasts approximately $8 \mathrm{~min}$. Block A targets precision stepping by practicing accurate foot placement on the projected stepping tiles. Block $\mathrm{B}$ targets obstacle negotiation by avoiding the projected obstacles. Block $\mathrm{C}$ elicits changes in the direction of progression by using a variety of slalom trajectories. Block D targets precision acceleration and deceleration, as the participants must walk within a projected square that moves forward and backward on the treadmill. Block $\mathrm{E}$ challenges walking at different walking speeds. Block $F$ is the endgame, a 5-min track that combines several gait adaptability components in an interactive way. All sessions end with a coolingdown. To further promote the level of variability, each training block consists of small components (i.e., for block A: Stepping Tiles: belt speed will momentarily increase; width between the stepping stones will momentarily decrease). In addition, different walking speeds are used: $100 \%$ is the participant's comfortable walking speed on the treadmill. This will be determined during the first training session. The belt speed will be manually increased until the participant experiences it as comfortable. The therapist will then increase the belt speed with $0.3 \mathrm{~m} / \mathrm{s}$ and slowly decrease the belt speed until the participant again experiences it as comfortable. The average of both speeds will be used to set the comfortable walking speed. Other percentages (e.g., 40\%, 70\%, 120\%) are derived from this reference speed. The C-Mill training will be carried out by a physiotherapist with C-Mill certification. Progression over the training period is initiated and controlled by this therapist and based on the patient's capacity and performance. It comprises of increasing the level of task variability, increasing obstacle size, and the addition of a dual task, for example the use of the auditory Stroop task. During the Stroop task, participants listen to an audiotape presenting a random sequence of the words "high" or "low", expressed in either a low-pitched voice or a high-pitched voice. They are asked to respond aloud indicating the pitch of the word ("high" or "low"), while ignoring the (randomly conflicting) semantic meaning of the word.

\section{Procedure and assessments}

All outcome measurements will be collected during the assessments at the movement laboratory (Table 1). The intervention group is evaluated three times: pre C-Mill training (week 16), post C-Mill training (week 22), and at the end of the follow-up (week 38). The control group is evaluated four times: pre waiting-list (week 16), post waiting-list (week 22), post C-Mill training (week 28), and at the end of the follow-up (week 44). The assessments will follow a standardized protocol and are conducted by the primary investigator (LV) who is trained to perform the outcome measurements. As the primary investigator takes part in the training sessions, outcome assessment cannot be blinded. During all assessments, the use of orthotic devices and/or orthopedic footwear is allowed depending on the task. No other (walking) aids are allowed. If participants use any orthotic or orthopedic 


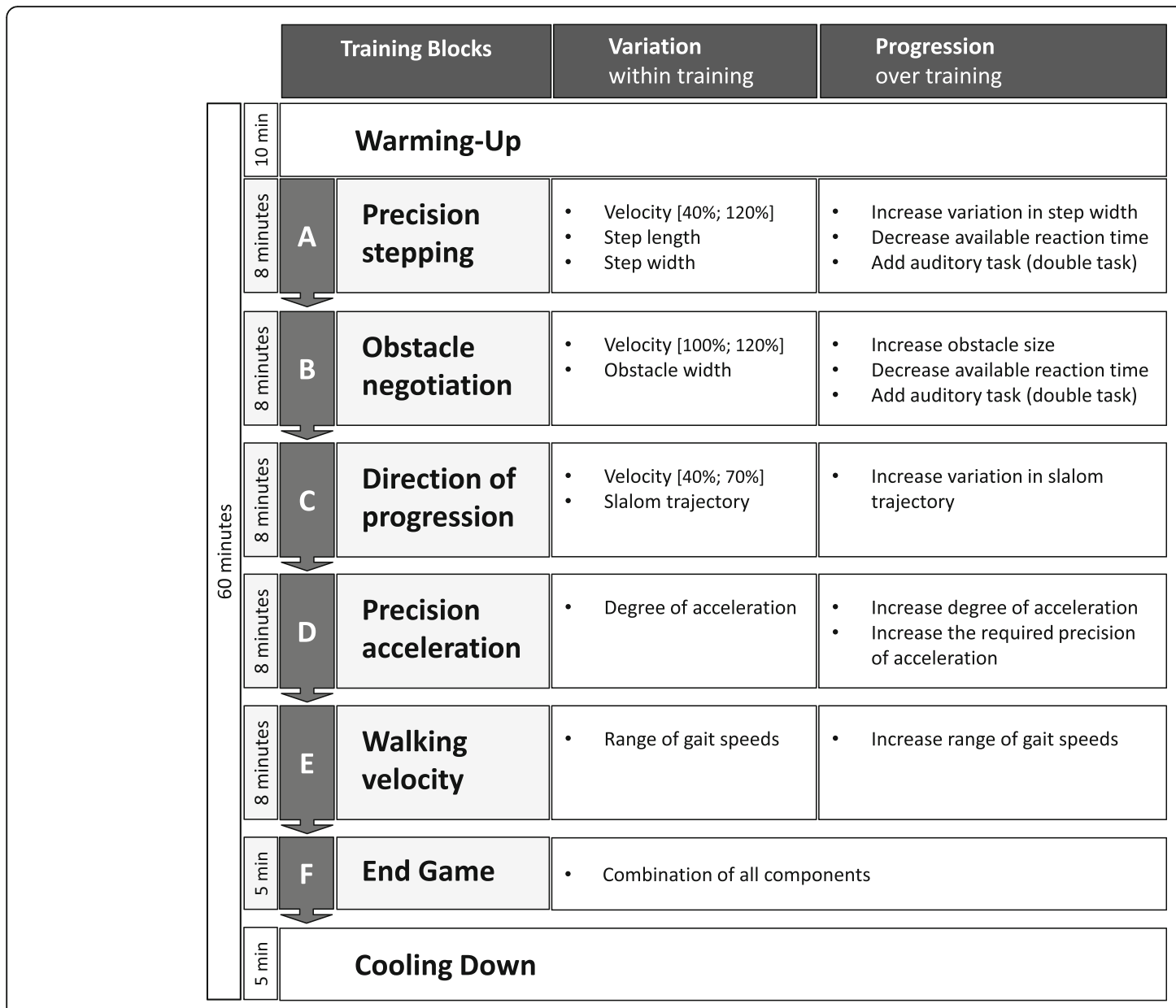

Fig. 2 Overview of the C-Mill training

device during a task, this will be registered and kept constant throughout the consecutive assessments.

\section{Demographic and clinical assessments}

The demographic and clinical assessments are collected during the first assessment in the movement laboratory. The demographic characteristics consist of age; sex; height; weight; presence (or absence) of a genetic diagnosis and inheritance pattern; disease duration (i.e., the number of years since symptom onset); regular use of medication, orthopedic shoes or orthotic devices, or other walking aids; presence and severity of visual deficits; and number of falls in the preceding year.

Clinical assessments consist of the Spastic Paraplegia Rating Scale (SPRS) to determine disease severity [19]. Bilateral muscle strength is scored with the Medical Research Council (MRC) scale for hip adduction, abduction, flexion, and extension; knee flexion and extension; and ankle plantar and dorsiflexion [4, 20,21]. Bilateral muscle tonus is scored with the Modified Ashworth Scale (MAS) for the hip adductors (hip $70^{\circ}$ flexed), knee flexion and extension, and ankle plantar and dorsiflexion with knee extended (gastrocnemius) and knee flexed (soleus) [22]. Vibration sense is evaluated using a tuning fork on bilateral patella, lateral malleolus, and at the first metatarsophalangeal joint. Trunk control is assessed using the Trunk Control Measurement Scale (TCMS) [23]. Lastly, coordination is examined via (i) toe tapping, and (ii) leg agility [24].

\section{Primary outcome}

The primary outcome is gait adaptability as measured with the obstacle subtask of the Emory Functional Ambulation Profile (E-FAP). Participants are asked to negotiate a 10-m course in which two wooden blocks (100(l) $\times 10(\mathrm{w}) \times 5(\mathrm{~h}) \mathrm{cm})$ and a bin are placed along the walkway. The instruction given is to complete the task as fast as possible but keep your own safety in mind. The number of seconds needed to complete the task is registered. A faster time score indicates better gait adaptability. The obstacle subtask of the E-FAP has previously been used as an outcome measure for gait adaptability in several neurological populations [14, 15]. The full protocol is available online and via Wolf et al. [25]. 
Table 1 Standard protocol items: Recommendations for interventional trials (SPIRIT) figure

\begin{tabular}{|c|c|c|c|c|c|c|}
\hline \multirow[b]{2}{*}{$\begin{array}{l}\text { TIMEPOINT in weeks } \\
\text { (intervention / usual care) }\end{array}$} & \multirow{2}{*}{$\begin{array}{c}\text { Enrollment } \\
\qquad-\mathrm{T}_{1}\end{array}$} & \multirow{2}{*}{$\begin{array}{c}\text { Allocation } \\
\qquad \begin{array}{c}T_{0}= \\
(0 / 0)\end{array}\end{array}$} & \multicolumn{3}{|c|}{ Post-allocation } & \multirow{2}{*}{$\begin{array}{c}\text { Follow-up } \\
\qquad \begin{array}{c}T_{3}= \\
(38 / 44)\end{array}\end{array}$} \\
\hline & & & $\begin{array}{c}\mathrm{T}_{1}= \\
(16 / 16)\end{array}$ & $\begin{array}{c}T_{2}= \\
(22 / 22)\end{array}$ & $\begin{array}{c}T_{S}= \\
(N A / 28)\end{array}$ & \\
\hline \multicolumn{7}{|l|}{ ENROLLMENT: } \\
\hline $\begin{array}{r}\text { Eligibility screen } \\
\text { Informed consent } \\
\text { Allocation }\end{array}$ & $\begin{array}{l}x \\
x\end{array}$ & $x$ & & & & \\
\hline \multicolumn{7}{|l|}{ INTERVENTION: } \\
\hline $\begin{array}{r}\text { Intervention program } \\
\text { Usual care }\end{array}$ & & & $\begin{array}{l}x \\
x\end{array}$ & $\begin{array}{l}x \\
x\end{array}$ & $x$ & $\begin{array}{l}x \\
x\end{array}$ \\
\hline \multicolumn{7}{|l|}{ ASSESSMENTS: } \\
\hline $\begin{array}{l}\text { Primary Outcome } \\
\qquad \begin{array}{r}\text { Emory functional ambulation } \\
\text { profile - obstacle subtask }\end{array}\end{array}$ & & & $\mathrm{x}$ & $\mathrm{x}$ & $x$ & $\mathrm{x}$ \\
\hline \multicolumn{7}{|l|}{ Secondary Outcome } \\
\hline 10 meter walk test & & \multirow{7}{*}{$x$} & $\mathrm{x}$ & $x$ & $x$ & $x$ \\
\hline Mini balance evaluation systems test & & & $\mathrm{x}$ & $x$ & $x$ & $x$ \\
\hline Daily levels of physical activity & & & $x$ & $x$ & $x$ & $x$ \\
\hline Activities-specific balance confidence scale & & & $x$ & $x$ & $x$ & $x$ \\
\hline Fall calendar & & & & & & $x$ \\
\hline Walking adaptability ladder test & & & $x$ & $x$ & $x$ & $x$ \\
\hline Spatio-temporal gait parameters & & & $x$ & $x$ & $x$ & $x$ \\
\hline \multicolumn{7}{|l|}{ Other parameters } \\
\hline Age & $\mathrm{x}$ & & & & & \\
\hline Presence (or absence) of genetic diagnosis & $x$ & & & & & \\
\hline $\begin{array}{r}\text { Medical history } \\
\text { Demographics: } \\
\text { (height, weight, use of medication, use of } \\
\text { orthopedic devices or walking aids, visual } \\
\text { deficits, number of falls in preceding year) }\end{array}$ & $x$ & & $x$ & & & \\
\hline Spastic Paraplegia Rating Scale & & & $x$ & & & \\
\hline Muscle strength & & & $x$ & & & \\
\hline Muscle tonus & & & $x$ & & & \\
\hline Vibration sense & & & $x$ & & & \\
\hline Trunk control measurement scale & & & $x$ & & & \\
\hline Coordination & & & $x$ & & & \\
\hline Co-interventions & & $x$ & $x$ & $x$ & $x$ & $x$ \\
\hline Adverse events & & $x$ & $x$ & $x$ & $x$ & $x$ \\
\hline
\end{tabular}

Overview of enrollment, interventions, and assessments during the study protocol. Timepoints $T_{1}, T_{2}$, and $T_{3}$ are applicable for participants in the intervention group and usual care group. Timepoint $T_{s}$ is only applicable for participants in the usual care group

NA not applicable 


\section{Secondary outcomes}

Secondary outcome measures comprise of the following clinical tests:

\section{- 10-Meter Walk Test (10MWT)}

The $10 M W T$ is a standardized and recommended measurement of walking velocity. Participants walk $13 \mathrm{~m}$ in a straight line, first three times at comfortable speed and then three times as fast as possible. Participants have $3 \mathrm{~m}$ to accelerate to the requested speed. When the first foot crosses the 3-m line, the timer starts. The timer stops when the first foot crosses the 13-m line. Like this, the number of seconds it takes to walk $10 \mathrm{~m}$ is recorded [26]. The test has been found reliable, valid, and sensitive in neurological populations [26] and has been used in people with HSP $[9,13,27,28]$.

\section{- Mini Balance Evaluation Systems Test (miniBEST)}

The mini-BEST is a 14-item, 3-point ordinal rating scale ( $0-2$ points) to evaluate balance capacity in 4 subcategories: anticipatory postural control, reactive postural control, sensory orientation, and gait stability. The attainable sum scores range from 0 to 28 points, a higher score indicating better balance capacity. Participants perform the test barefoot. The full protocol is available online and has been described by Franchignoni et al. [29]. The mini-BEST is often used in neurological populations; has been found valid, reliable, and responsive [3032]; and has been recommended for use in people with HSP [4].

- Physical activity levels during daily life

Physical activity during daily life will be registered via the Activ8 Physical Activity Monitor (Activ8, Remedy Distribution Ltd., Valkenswaard, The Netherlands). The Activ8 monitor is a small $(30 \times 32 \times 10 \mathrm{~mm})$ and lightweight device with three axial accelerometers. It registers body positions (non-wear of the Activ8/lying, sitting, and standing) and activities (walking, running, cycling) [33]. The Activ8 is placed by the primary researcher using Tegaderm ${ }^{\mathrm{Tm}}$ tape on the right upper thigh of the participants. Interval for data sampling will be set to one measurement per $15 \mathrm{~s}$. Collected measures consist of total time spent walking (minutes) and total time spent active (i.e., minutes classified as walking, running or cycling).

\section{- Activities-specific Balance Confidence scale (ABC)}

Balance confidence will be measured using the ABC. The questionnaire describes sixteen indoor and outdoor situations. Participants are asked to express their confidence in safely executing the proposed situations without falling. Scores range from 0 to 100 , a higher score indicating more confidence. The $\mathrm{ABC}$ has been used to assess balance confidence in people with HSP [27, 34]. The full questionnaire is available via Powell et al. [35].

\section{- Fall calendar}

The fall calendar is used to monitor falls and near falls and is self-reported by the participants during a 15-week period. The World Health Organization defined a fall as "an event which results in a person coming to rest inadvertently on the ground or other lower level." A near fall is defined as "a stumble event or loss of balance that would result in a fall if sufficient recovery mechanisms were not activated" [36]. In addition, participants register incidents where a fall was likely to happen, but was averted through the action of another person. When a (near) fall occurs, the participant is asked to report a short description of the event, the environment (indoor/ outdoor, illuminated/dark space, and surface (e.g., tiles, carpet, forest)), and lastly, whether the (near) fall resulted in any injuries. To meet participants' preference, calendars can be filled in digitally or on paper. Every other week, participants are reminded of the fall calendar via a phone call from the primary investigator (LV).

\section{- Walking Adaptability Ladder Test (WALT)}

The Walking Adaptability Ladder Test (WALT) is a test to measure step precision. A standardized ladder is placed on the floor. It consists of 17 rectangular stepping targets that gradually decrease $2 \mathrm{~cm}$ in length (range 64-32 cm). Participants start stepping in the largest target and walk as fast as possible to the other side, turn and hit the targets in reverse order while avoiding the ladder rungs. The instructions are to perform the test as fast as possible, but try to prevent foot placement errors. The test is timed: a faster time is indicative of better stepping precision. Participants perform the test four times, first twice with one foot per target and, thereafter, twice with both feet in one target. A time penalty of 0.5 $\mathrm{s}$ is added each time a participant makes a foot placement mistake.

\section{- Spatio-temporal gait parameters}

Spatio-temporal gait parameters are collected with a 3D full body gait analysis using Vicon (Vicon` Motion systems Ltd.) at the movement laboratory of the Radboudumc, Nijmegen. Retroreflective markers are placed on anatomical landmarks according to the standard Plug-in Gait marker model for upper and lower 
body. In addition, participants will wear accelerometers on their lateral heels, as the higher measuring frequency will enable a more accurate gait event detection. Participants will walk two bouts of 3 min over an 8-m walkway. The following spatio-temporal parameters will be retrieved: step length $(\mathrm{cm})$, step width $(\mathrm{cm})$, step time $(\mathrm{s})$, walking speed $(\mathrm{m} / \mathrm{s})$, stride time $(\mathrm{s})$, stride length $(\mathrm{cm})$, and cadence (steps/min).

\section{Assessment of therapy adherence and co-interventions}

To support adherence to the protocol, participants will be in direct contact with the primary investigator (LV) by telephone every other week. This enables the investigator to verbally confirm assessments and training dates, check adherence to the fall calendar, and quickly address and resolve questions and possible problems that may interfere with continuation of the protocol. In addition, participants are offered flexible time slots for the assessments and training sessions. Assessment of adherence to the C-Mill protocol is possible as therapists will log the performed C-Mill trainings. In case of an unexpected cancelation, the reason will be registered, and the missed training can be compensated in the next week. When multiple consecutive training sessions cannot proceed, a pragmatic solution is sought so that the participant is able to complete the protocol.

In addition, assessment of co-intervention will take place during the assessments. Participants are asked to self-report in a survey what type of co-intervention they received (e.g., physical therapy, occupation therapy).

\section{Sample size}

Sample size calculation is based on previous studies assessing effectiveness of C-Mill training on the obstacle subtask of the E-FAP scores in neurological populations $[14,15]$. A total of 32 participants is sufficient to demonstrate an improvement on the E-FAP score of $1.75 \mathrm{~s}$ ( $\mathrm{SD}=2.0 \mathrm{~s}, \alpha=0.05, \beta=0.2$ ). Considering a $10 \%$ attrition rate, 36 participants will be included.

\section{Statistical analysis}

The effect of gait adaptability training on primary and secondary outcomes will be tested using ANCOVA. Postintervention measurements will be used as dependent variables with pre-intervention measurements as the covariate. Group (C-Mill versus waiting list) is used as an independent between-subjects factors. The retention of gait adaptability training will be tested by merging both groups and using a repeated measures ANOVA with time as a within-subjects factor (C-Mill group: assessments 1 , 2 , and 3; waiting list: assessments 2, 3, and 4). Post hoc tests will be performed in the case of significant main or interaction effects, using paired $t$ tests. Fall rates will be processed descriptively. Depending on the distribution of the data, the rate of near falls may be analyzed using Wilcoxon signed rank test. In addition, to determine key determinants of C-Mill training efficacy, a stepwise linear regression analysis will be performed with traininginduced change in gait adaptability (relative change of the obstacle subtask of the E-FAP) as the dependent variable. Univariate analyses will be performed to select the best factors from the available demographic and clinical characteristics.

\section{Discussion}

Limitations in walking capacity are among the most disabling symptoms in people with hereditary spastic paraplegia (HSP). A handful of studies aimed to improve walking capacity in people with $\operatorname{HSP}[9,13$, 37], but these studies did not include context-specific exercises aimed at gait adaptability. Gait adaptability has been successfully trained in several neurological populations using augmented reality on the C-Mill $[14,15]$, but so far, this has never been done in people with HSP.

Move-HSP is a two-armed, open-label randomized controlled trial that will be the first study to assess the effects of gait adaptability training in people with pure HSP. Participants in the intervention group receive $10 \mathrm{~h}$ (1-h sessions; twice per week) of protocolled C-Mill training, whereas the control group continues with treatment as usual (waiting list). After 5 weeks on the waiting list, the control group will cross over and follow gaitadaptability training. The primary outcome is gait adaptability assessed with the obstacle subtask of the E-FAP. Secondary outcomes focus on several aspects of balance and gait capacity. Mildly to moderately affected people with pure HSP that fit the a-priori established eligibility criteria will be included. There are no restrictions regarding sex, symptom duration, or use of orthotic/orthopedic devices in order to represent the clinical heterogeneity characteristic of people with pure HSP. Yet, to provide proof op principle and limit the influence of impaired cognitive capacity, people with complex forms of HSP are excluded.

Move-HSP aims to make a step towards evidencebased and individually tailored gait rehabilitation programs for people with HSP. It will reveal whether context-specific training is an effective tool for improving gait adaptability in people with pure HSP. If the CMill intervention results in improved walking adaptability, it may be beneficial to implement this type of training on a regular basis in the rehabilitation of people with HSP. In addition, knowledge of the key determinants of training efficacy will help to optimize the selection of subjects with HSP that are most responsive to gait adaptability training. 


\section{Trial status}

Participant recruitment has started in November 2019 and is currently ongoing. So far, no assessments in the movement laboratory have yet been conducted. It was originally anticipated that a total of 10 months would be needed to complete the recruitment and 24 months to complete the entire protocol, but the current SARSCoV-2 pandemic will undoubtedly lengthen these periods to an unforeseeable extent. This study protocol is based on protocol version 5, dated October 31, 2019. An amendment to add training locations was approved on February 18, 2020.

\section{Supplementary information}

Supplementary information accompanies this paper at https://doi.org/10. 1186/s13063-020-04932-9.

Additional file 1. (MP4 $9073 \mathrm{~kb})$

\section{Abbreviations}

10MWT: 10 Meter Walk Test; ABC: Activities-specific Balance Confidence Scale; BTX: Botulinum toxin; E-FAP: Emory Functional Ambulation Profile; ERN-RND: European Reference Network for Rare Neurological Diseases: HSP: Hereditary spastic paraplegia; MAS: Modified Ashworth Scale; miniBEST: Mini Balance Evaluation Systems Test; MRC: Medical Research Council; Radboudumc: Radboud University Medical Center; SPRS: Spastic Paraplegia Rating Scale; TCMS: Trunk Control Measurement Scale; WALT: Walking Adaptability Ladder Test

\section{Acknowledgements}

Several authors (BW, AG, JN) of this publication are members of the European Reference Network for Rare Neurological Diseases- Project ID No 739510. We wish to acknowledge Noël Keijsers, PhD, for his expert advice on this project.

\section{Safety}

The risk associated with participation will be negligible, according to the risk classification of the NFU. In case of an adverse event, it will be recorded and reported to the accredited METC and monitored until it has abated, or until a stable situation has been reached. Both sponsor and investigator are insured in accordance with the legal requirements in the Netherlands. Moreover, the investigator can decide to withdraw participants from the study for urgent medical reasons.

\section{Data management and monitoring}

Collected data will be processed with absolute discretion. Each participant receives an unidentifiable numerical code. A codelist to link subject to the numerical code is held separately from the data and is safeguarded by the investigators. The collected data will be entered into Castor EDC, an electronic data management system (www.castoredc.com). Handling of data will comply with the Dutch Personal Data Protection Act. A monitor is appointed, and monitoring is executed according to the Netherlands Federation of University Medical Centres (NFU) guidelines for negligible risk intensity. This process is independent from investigators and sponsors. After completion of the study, data will be checked, locked, and stored for 15 years in accordance with Dutch national legislation.

\section{Dissemination}

The results will be presented at (inter) national conferences, published in (inter) national scientific peer-reviewed journals, and included in the doctoral dissertation of LV.

\section{Authors' contributions}

The first version of Move-HSP was drafted by JN, BW, and AG. The acquisition of funding from The Gossweiler Foundation was led by JN, BW, and AG. The protocol was finalized with the contributions of JN, BW, AG, WW, BL, and LV. All authors contributed to the refinement and approved the final manuscript.

\section{Funding}

The study is funded by the Jacques und Gloria Gossweiler Foundation. A grant from Ipsen Pharmaceuticals supports the costs of the physiotherapists delivering the training. The funders are not involved in the development of the protocol, nor are they involved in the execution, analyses, or interpretation of the study data.

\section{Availability of data and materials}

Not applicable.

\section{Ethics approval and consent to participate}

Ethical approval for the study protocol has been obtained from the accredited medical ethical committee Arnhem-Nijmegen, the Netherlands (2019-5602, NL70295.091.19). The committee will be notified of any protocol modification via an amendment. In addition, the trial has been registered on Clinicaltrials.gov (NCT04180098). Informed consent is signed and dated by all participants prior to data collection and obtained by the primary investigator (LV) during a home visit.

\section{Consent for publication}

Not applicable.

\section{Competing interests}

The authors declare that they have no competing interests.

\section{Author details}

${ }^{1}$ Department of Rehabilitation; Center of Expertise for Parkinson \& Movement Disorders, Donders Institute for Brain, Cognition and Behavior; Radboud University Medical Center, PO Box 9101, 6500 HB Nijmegen, The Netherlands. ${ }^{2}$ Department of Neurology; Center of Expertise for Parkinson \& Movement Disorders, Donders Institute for Brain, Cognition and Behavior; Radboud University Medical Center, Nijmegen, The Netherlands. ${ }^{3}$ Department of Rehabilitation, Sint Maartenskliniek, Ubbergen, The Netherlands.

Received: 28 May 2020 Accepted: 25 November 2020

Published online: 07 January 2021

\section{References}

1. Salinas S, Proukakis C, Crosby A, Warner $\Pi$ T. Hereditary spastic paraplegia: clinical features and pathogenetic mechanisms. Lancet Neurol. 2008;7(12): 1127-38.

2. Harding AE. Classification of the hereditary ataxias and paraplegias. Lancet. 1983;1 (8334):1151-5.

3. McDermott C, White K, Bushby K, Shaw P. Hereditary spastic paraparesis: a review of new developments. J Neurol Neurosurg Psychiatry. 2000;69(2): 150-60.

4. Nonnekes J, van Lith B, van de Warrenburg BP, Weerdesteyn V, Geurts ACH. Pathophysiology, diagnostic work-up and management of balance impairments and falls in patients with hereditary spastic paraplegia. J Rehabil Med. 2017:49(5):369-77.

5. Harding AE. Hereditary spastic paraplegias. Semin Neurol. 1993;13(4):333-6.

6. Marsden J, Ramdharry G, Stevenson V, Thompson A. Muscle paresis and passive stiffness: key determinants in limiting function in hereditary and sporadic spastic paraparesis. Gait \& posture. 2012;35(2):266-71.

7. Shribman S, Reid E, Crosby AH, Houlden H, Warner TT. Hereditary spastic paraplegia: from diagnosis to emerging therapeutic approaches. Lancet Neurol. 2019;18(12):1136-46.

8. Klimpe S, Schule R, Kassubek J, Otto S, Kohl Z, Klebe S, et al. Disease severity affects quality of life of hereditary spastic paraplegia patients. Eur J Neurol. 2012:19(1):168-71.

9. Bertolucci F, Di Martino S, Orsucci D, lenco EC, Siciliano G, Rossi B, et al. Robotic gait training improves motor skills and quality of life in hereditary spastic paraplegia. NeuroRehabil. 2015;36(1):93-9.

10. Nonnekes J, de Niet M, Oude Nijhuis LB, de Bot ST, van de Warrenburg BP, Bloem BR, et al. Mechanisms of postural instability in hereditary spastic paraplegia. J Neurol. 2013;260(9):2387-95.

11. van Lith BJH, Kerstens $H$, van den Bemd LAC, der Sanden M, Weerdesteyn V Smeets $R$, et al. Experienced complaints, activity limitations and loss of 
motor capacities in patients with pure hereditary spastic paraplegia: a webbased survey in the Netherlands. Orphanet J Rare Dis. 2020;15(1):64.

12. Kerstens H, Satink T, Nijkrake MJ, De Swart BJM, Van Lith BJH, Geurts ACH, et al. Stumbling, struggling, and shame due to spasticity: a qualitative study of adult persons with hereditary spastic paraplegia. Disabil Rehabil. 2020; 42(26):3744-51.

13. Seo HG, Oh BM, Kim K. Robot-assisted gait training in a patient with hereditary spastic paraplegia. PM R. 2015;7(2):210-3.

14. Heeren A, van Ooijen M, Geurts AC, Day BL, Janssen TW, Beek PJ, et al. Step by step: a proof of concept study of C-Mill gait adaptability training in the chronic phase after stroke. J Rehabil Med. 2013;45(7):616-22.

15. Fonteyn EM, Heeren A, Engels JJ, Boer JJ, van de Warrenburg BP, Weerdesteyn V. Gait adaptability training improves obstacle avoidance and dynamic stability in patients with cerebellar degeneration. Gait Posture. 2014;40(1):247-51.

16. Peruzzi A, Zarbo IR, Cereatti A, Della Croce U, Mirelman A. An innovative training program based on virtual reality and treadmill: effects on gait of persons with multiple sclerosis. Disabil Rehabil. 2017;39(15):1557-63.

17. Kaji R, Osako Y, Suyama K, Maeda T, Uechi Y, Iwasaki M. Botulinum toxin type A in post-stroke lower limb spasticity: a multicenter, double-blind, placebo-controlled trial. J Neurol. 2010;257(8):1330-7.

18. Wissel J, Ward AB, Erztgaard P, Bensmail D, Hecht MJ, Lejeune TM, et al. European consensus table on the use of botulinum toxin type $A$ in adult spasticity. J Rehabil Med. 2009;41(1):13-25.

19. Schule R, Holland-Letz T, Klimpe S, Kassubek J, Klopstock T, Mall V, et al. The Spastic Paraplegia Rating Scale (SPRS): a reliable and valid measure of disease severity. Neurology. 2006;67(3):430-4.

20. Counsil MR. Aids to the investigation of the peripheral nervous system. London. Her Majesty's Stationary Office. 1943.

21. Dyck PJ, Boes CJ, Mulder D, Millikan C, Windebank AJ, Dyck PJ, et al. History of standard scoring, notation, and summation of neuromuscular signs. A current survey and recommendation. J Peripheral Nervous Syst. 2005;10(2): 158-73.

22. Bohannon RW, Smith MB. Interrater reliability of a modified Ashworth scale of muscle spasticity. Phys Ther. 1987;67(2):206-7.

23. Heyrman L, Molenaers G, Desloovere K, Verheyden G, De Cat J, Monbaliu E, et al. A clinical tool to measure trunk control in children with cerebral palsy: the Trunk Control Measurement Scale. Res Dev Disabil. 2011:32(6):2624-35.

24. Martinez-Martin P, Rodriguez-Blazquez C, Alvarez-Sanchez M, Arakaki T, Bergareche-Yarza A, Chade A, et al. Expanded and independent validation of the Movement Disorder Society-Unified Parkinson's Disease Rating Scale (MDS-UPDRS). J Neurol. 2013;260(1):228-36.

25. Wolf SL, Catlin PA, Gage K, Gurucharri K, Robertson R, Stephen K. Establishing the reliability and validity of measurements of walking time using the Emory Functional Ambulation Profile. Phys Ther. 1999;79(12): 1122-33.

26. Graham JE, Ostir GV, Fisher SR, Ottenbacher KJ. Assessing walking speed in clinical research: a systematic review. J Eval Clin Pract. 2008;14(4):552-62.

27. van Lith BJH, den Boer J, van de Warrenburg BPC, Weerdesteyn V, Geurts AC. Functional effects of botulinum toxin type $A$ in the hip adductors and subsequent stretching in patients with hereditary spastic paraplegia. J Rehabil Med. 2019;51(6):434-41.

28. Denton AL, Hough AD, Freeman JA, Marsden JF. Effects of superficial heating and insulation on walking speed in people with hereditary and spontaneous spastic paraparesis: a randomised crossover study. Ann Phys Rehabil Med. 2018;61(2):72-7.

29. Franchignoni F, Horak F, Godi M, Nardone A, Giordano A. Using psychometric techniques to improve the Balance Evaluation Systems Test: the mini-BESTest. J Rehabil Med. 2010;42(4):323-31.

30. Leddy AL, Crowner BE, Earhart GM. Functional gait assessment and balance evaluation system test: reliability, validity, sensitivity, and specificity for identifying individuals with Parkinson disease who fall. Phys Ther. 2011;91(1): 102-13.

31. Ross E, Purtill H, Uszynski M, Hayes S, Casey B, Browne C, et al. Cohort study comparing the Berg Balance Scale and the Mini-BESTest in people who have multiple sclerosis and are ambulatory. Phys Ther. 2016;96(9):1448-55.

32. Tsang CS, Liao LR, Chung RC, Pang MY. Psychometric properties of the Mini-Balance Evaluation Systems Test (Mini-BESTest) in community-dwelling individuals with chronic stroke. Phys Ther. 2013;93(8):1102-15.

33. Valkenet $\mathrm{K}$, Veenhof $\mathrm{C}$. Validity of three accelerometers to investigate lying, sitting, standing and walking. PLoS One. 2019;14(5):e0217545.
34. de Niet M, de Bot ST, van de Warrenburg BP, Weerdesteyn V, Geurts AC Functional effects of botulinum toxin type-A treatment and subsequent stretching of spastic calf muscles: a study in patients with hereditary spastic paraplegia. J Rehabil Med. 2015;47(2):147-53.

35. Powell LE, Myers AM. The Activities-specific Balance Confidence (ABC) Scale. J Gerontol A Biol Sci Med Sci. 1995;50a(1):M28-34.

36. Faber I, Martinez ARM, Martins CR Jr, Maia ML, Souza JP, Lourenco CM, et al. SPG11-related parkinsonism: clinical profile, molecular imaging and I-dopa response. Mov Disord. 2018;33(10):1650-6.

37. Zhang Y, Roxburgh R, Huang L, Parsons J, Davies TC. The effect of hydrotherapy treatment on gait characteristics of hereditary spastic paraparesis patients. Gait Posture. 2014;39(4):1074-9.

\section{Publisher's Note}

Springer Nature remains neutral with regard to jurisdictional claims in published maps and institutional affiliations.
Ready to submit your research? Choose BMC and benefit from:

- fast, convenient online submission

- thorough peer review by experienced researchers in your field

- rapid publication on acceptance

- support for research data, including large and complex data types

- gold Open Access which fosters wider collaboration and increased citations

- maximum visibility for your research: over $100 \mathrm{M}$ website views per year

At BMC, research is always in progress.

Learn more biomedcentral.com/submissions 